BMJ Open

Sport \&

Exercise

Medicine

\section{Vibrotactile feedback as a tool to improve motor learning and sports performance: a systematic review}

To cite: van Breda $E$, Verwulgen S, Saeys W, et al. Vibrotactile feedback as a tool to improve motor learning and sports performance: a systematic review. BMJ Open Sport Exerc Med 2017;3:e000216. doi:10.1136/bmjsem-2016000216

EB and SV contributed equally.

Accepted 3 May 2017
CrossMark

\footnotetext{
${ }^{1}$ Faculty of Medicine and Health Sciences, Department of Rehabilitation Sciences and Physiotherapy (MOVANT), University of Antwerp, Antwerp, Belgium ${ }^{2}$ Product Development, Faculty of Design Sciences, University of Antwerp, Antwerp, Belgium
}

Correspondence to Dr Eric van Breda; eric. vanbreda@uantwerpen.be

\section{ABSTRACT \\ Background Evidence concerning the use of} vibrotactile feedback for acquiring and learning new motor skills is limited. Although various concepts and applications for tactile feedback have been proposed, little is known about the suitability of this feedback mechanism in sports training.

Aim The goal of this systematic review was to gather knowledge on the efficacy of the use of vibrotactile feedback in improving sports performance skills.

Design Systematic review.

Methods Comprehensively searched databases were: PubMed, Cochrane and Web of Science. Studies investigating the effects of using vibrotactile feedback in sports training in healthy subjects were included in this review.

Results No consensus was found regarding the positive effectiveness on performance using vibrotactile feedback in a sports context. No evidence was found that the addition of tactile feedback is effective for acquiring new motor skills. None of the studies show a significant learning effect.

\section{INTRODUCTION}

When an athlete performs a designated movement (motor task), any attempt is neurophysiological evaluated by scanning the information originating from intrinsic feedback (IFB) systems such as visual, auditory, proprioceptive and/or tactile feedback (TFB). Those feedback (FB) systems analyse and adjust extremity position either in real time (concurrent FB) or store the information during post-task evaluation (terminal FB). ${ }^{1}$ Thus IFB leads to the information regarding the outcome of the motor task (knowledge of results) and provides information of the entire process of a motor task (knowledge of performance). ${ }^{1}{ }^{2}$ The only IFB system that is in direct contact with the environment is tactile sensory system, known as TFB. TFB allows us to interact with the environment through specific sensors in the skin. They sense vibration, pressure, touch, texture and so on. TFB is complex and is primarily

\section{What is already known?}

Motor tasks are controlled by intrinsic feedback mechanisms.

- Augmented feedback (verbal instructions) in learning new motor tasks is important in sports.

\section{What are the new findings?}

- The use of vibrotactile feedback in several medical and non-medical areas has well been established.

- Vibrotactile feedback as feedback tool in sports has not yet been supported by scientific evidence.

- There lies a huge potential for sports science to study feedback mechanisms by using vibrotactile feedback as intervention tool.

composed of a skin sensation, enabling subjects to localise and recognise individual tactile cues and collaborates closely with the proprioceptive and kinaesthetic system, that is, body part position recognition. Interestingly, $\mathrm{TFB}$ is the only $\mathrm{FB}$ system that is bidirectional, that is, it interacts with the environment and simultaneously senses and processes such interactions. ${ }^{4-6}$ Although TFB is essential for movementcontrolled actions, it can also be a disturbing factor by creating a kind of sensory overload mainly because it can also be handled consciously. ${ }^{7}$

In contrast to IFB, augmented feedback (AFB) serves as an add-on to the taskintrinsic information and is exclusively delivered by external cues. ${ }^{2}$ To date, it is impossible to consider modern sports training, but also rehabilitation of certain patient groups, without the use of AFB. AFB comes from external sources, for example, trainers/coaches, electronic devices (displays, sounds), spectators and 
Table 1 PICOS and eligibility criteria

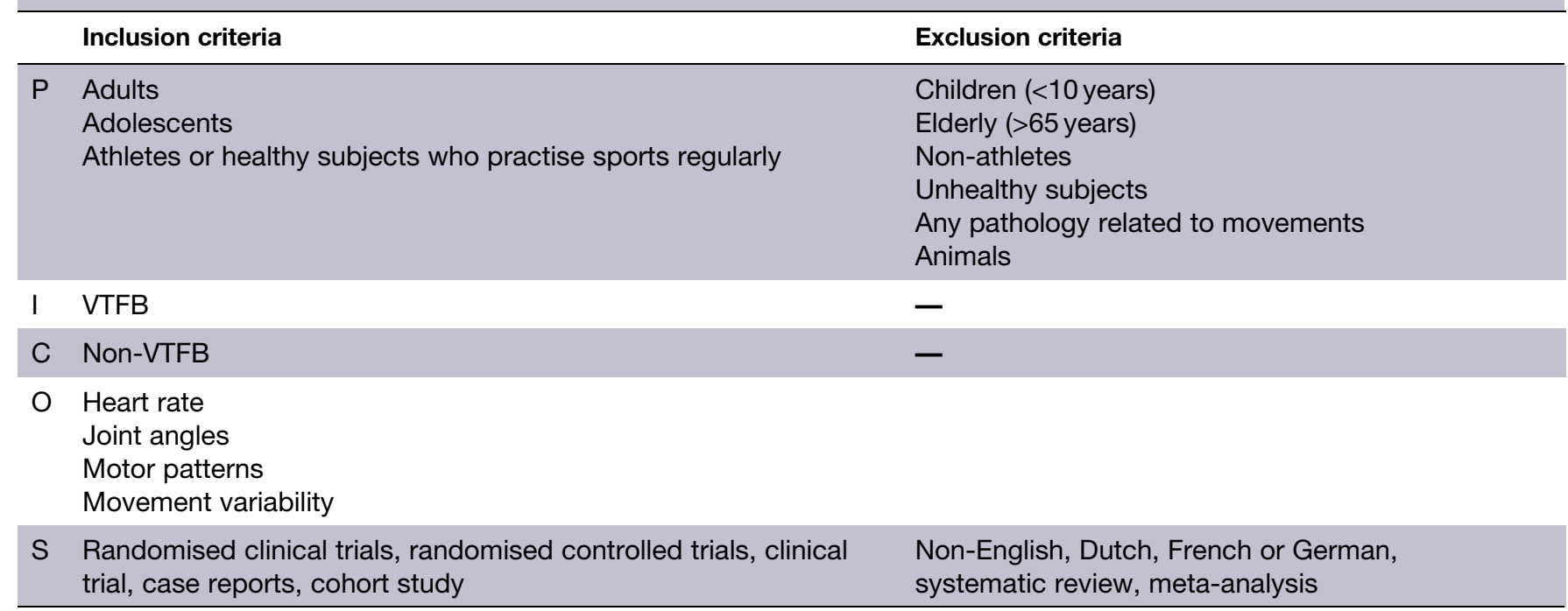

VTFB, vibrotactile feedback.

recently also haptic systems (robots, vibrotactile (VT) actuators), or a combination thereof. ${ }^{3}$ AFB has also been shown to play an important role in learning new motor tasks by enhancing the effect of IFB. ${ }^{5}{ }^{8}$ This can be provided verbally (a coach provides detailed spoken instructions), visually (a coach demonstrates the specific task) or haptic (a coach guides part of the trajectory of the movement by touching the subject at key parts of the motor task) cues. Mostly, however, AFB is offered after completion of a motor task, as terminal FB. Although it has been shown that terminal $\mathrm{FB}$ can play an important role in the learning process of motor tasks, the major drawback of this type of $\mathrm{FB}$ is that it only provides information on the outcome of the motor task but not real time on the process as in concurrent $\mathrm{FB}^{5}$ Nowadays, technological progress allows us to provide information to an athlete in real time. One of such methods is vibrotactile feedback (VTFB). During VTFB, a signal is generated by an actuator that applies vibrational stimuli to the skin guided by information on position of an extremity. ${ }^{9}$ VTFB is a form of concurrent FB and information can be delivered by varying temporal parameters in frequency, amplitude, waveform and/or duration. ${ }^{10}$ The tight coupling between motor task and the resulting VTFB stimuli requires a minimal latency. ${ }^{11}$ Instant and real-time augmented VTFB makes it feasible to provide spatial proprioceptive information during a complex motor task. ${ }^{12}$ In this sense, VTFB can thus be used to guide a motor task and provide instant FB. VTFB is, from a neurophysiological point of view, an interesting source of $\mathrm{FB}$, since it can bypass the athletes' visual FB system. The relatively small, lowweight and wireless potential makes it an excellent FB tool. ${ }^{3}$
Prior to the use of VTFB, consistent metaphors need to be learnt. Amemiya et al suggested that wearable VTFB devices should create realistic sensations. ${ }^{13}$ In that light, Spelmezan and Ziefle distinguished two types of encoding metaphors: a push and a pull metaphor. ${ }^{14}$ The pull metaphor means that the extremity of a subject reacts to a signal by moving towards the stimulus, whereas the push metaphor means that it pulls away from the stimulus.

The major aim of this present systematic review is to evaluate the definitions, methodologies and use of FB systems for learning motor tasks in sports. To the best of our knowledge, this is the first systematic review on VTFB systems within sports.

\section{METHODS \\ Design}

This systematic review was conducted in accordance with the PRISMA (Preferred Reporting Items for Systematic Reviews and Meta-Analyses) guidelines, which is an updated statement addressing the conceptual and methodological issues of the original QUOROM (Quality of Reporting of MetaAnalyses) statement. ${ }^{15}$

\section{Search strategy}

$\mathrm{PICO}(\mathrm{S})$ components were used to establish the research question. This $\mathrm{PICO}(\mathrm{S})$, along with the corresponding eligibility criteria, is shown in table 1.

To complete this review, three electronic databases were used. PubMed (http://www.ncbi.nlm.nih.gov/ pubmed/), Web of Science (WOS; https://webofknowledge.com/) and the Cochrane database (http:// www. cochranelibrary.com) were searched for peer-reviewed articles. 


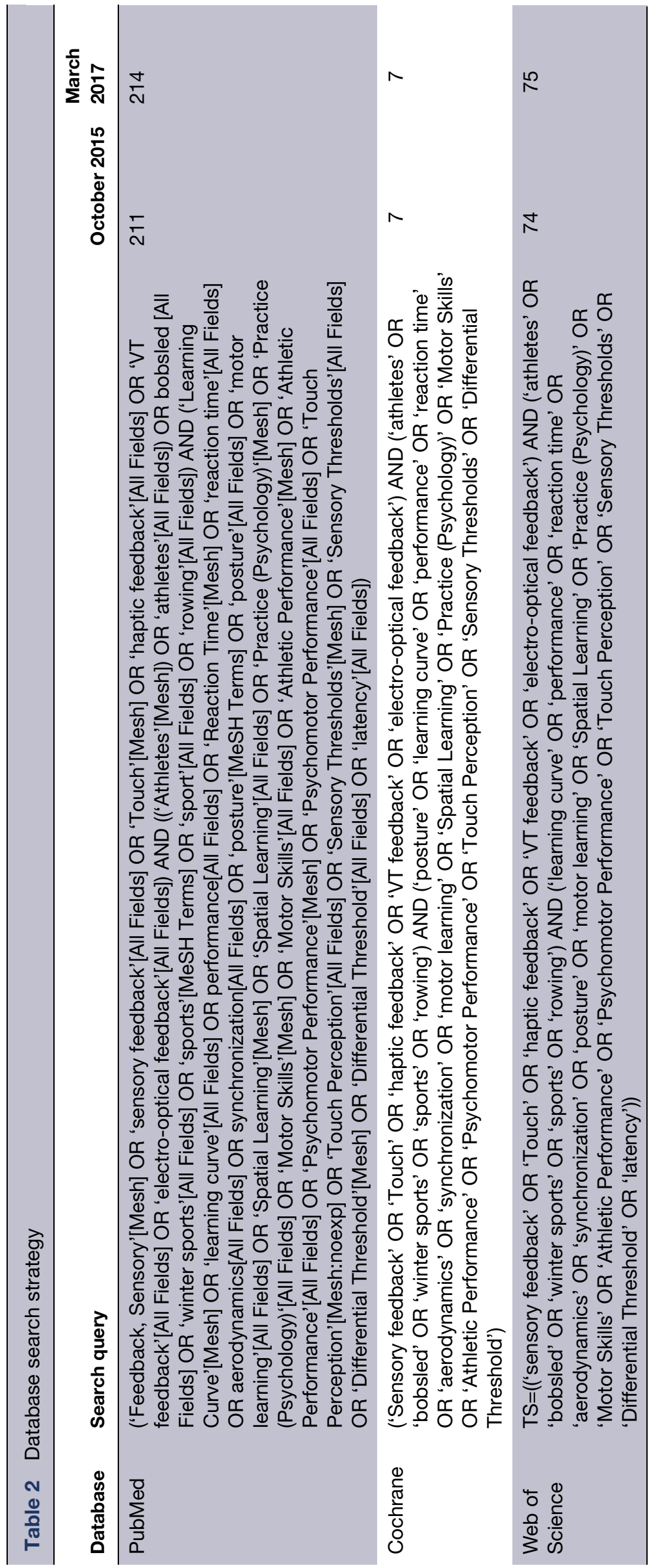


Table 3 EBRO classification of study results and recommendations: classification of the study results according to the level of evidence

A1 Meta-analyses (systematic reviews) which include at least some randomised clinical trials at quality level A2 that show consistent results between studies

A2 Randomised clinical trials of a good methodological quality (randomised double-blind controlled studies) with sufficient power and consistency

B Randomised clinical trials of a moderate methodological quality or with insufficient power, or other non-randomised, cohort or patient-control group study designs that involve intergroup comparisons

\section{Patient series \\ D Expert opinion}

EBRO, Evidence-Based Richtlijn(guideline)Ontwikkeling(development).

The Cochrane was used to find relevant clinical trials without date restrictions as search option. Using the advanced search option, a selection was made to find clinical trials only. Conference papers were excluded. FB conditions where VTFB is used to only robotically guide someone, that is, the subject playing a passive role, were also excluded from this review, along with FB conditions where VTFB is used as sensory substitution.

In addition, we manually searched for relevant articles based on article citations and reference lists in the PubMed and WOS databases. The articles found were extracted and organised in EndNote Online. Duplicates were removed manually. The first search was performed in October 2015 and the date of the last search was on 30 March 2017.

The search strategy is shown in table 2 .

\section{Study selection}

After implementation of the research strategy in all databases and the execution of the manual search, articles were extracted and organised in EndNote Online. Duplicates were deleted by hand. The remaining articles were transferred to an Excel template published by the KCE-Belgian Health Care Knowledge Centre (http://kce.fgov.be/). Two hundred and ninety-six (296) articles from the three used databases, 321 studies selected for screening and 25 records from other sources (30 March 2017) were sequentially included or excluded according to the eligibility criteria. Two reviewers (KW and TP) performed eligibility assessment independently in an unblinded standardised manner.

Thus, the inclusion criteria were:

1. athletes or healthy subjects who practise sports regularly;

2. VTFB or haptic FB;

3. heart rate (HR), joint angles, motor patterns, movement variability;

4. randomised clinical trials, randomised controlled trials, clinical trial, case reports, cohort study, and so on;
5. full text written in English, Dutch, German and French;

6. study subjects are adolescents or adults.

Exclusion criteria were:

1. non-healthy or pathological population and animals;

2. children $(<10$ years of age) and elderly $(>65+)$;

3 . other languages than those mentioned above;

4. meta-analysis, systematic reviews.

Experiments by Pressley and Ghatala indicated that children younger than 10 years of age are less accurately aware of FB results. Consequently, this set our lower age limit in our search strategy. ${ }^{16}$ The same goes for the upper limit in adults as has been shown by Rodrigue et al. ${ }^{17}$

The systematic screening of the articles was carried out in two different phases. First, all search results were screened based on title and abstract in a preliminary screening. If citations were considered potentially eligible and relevant, and if both reviewers (KW and TP) independently had agreed, full-text articles were obtained. In the second phase, full-text articles were evaluated. Detailed evaluation of the full-text publication was necessary for 47 articles. Disagreements between reviewers were resolved by consensus. If no consensus could be reached, a third reviewer (SV) would decide.

\section{Data extraction}

Two reviewers (KW and TP) independently performed data extraction by selecting relevant data and integrating it into two databases. The two databases were compared and integrated into a final extraction table. Again, disagreements were resolved through discussion between $\mathrm{KW}$ and $\mathrm{TP}$, and in case of disagreement a third author (SV) was asked to make a final judgement. None of the authors were contacted for further information when information was missing or unclearly reported. The reason for the latter was that non-peerreviewed unpublished information would, among others, hamper the systematic review process and could affect the quality of this paper as such. 


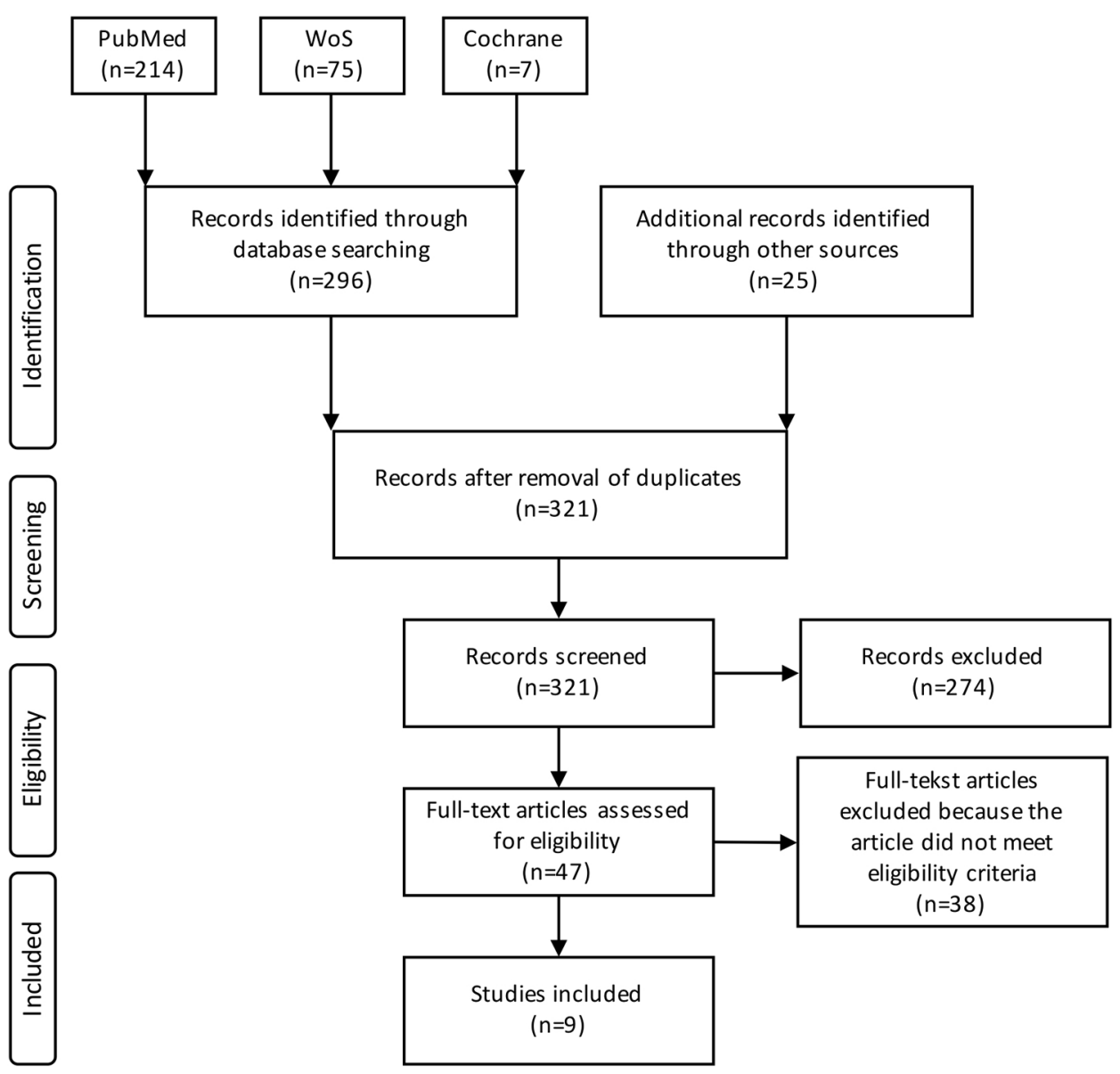

Figure 1 Flow chart of the search strategy and study selection process. WOS, Web of Science.

Items extracted from the studies were study design, age and number of subjects included, study characteristics (including branch of sports or movement studied, setting and FB conditions), VTFB conditions (including number of vibrotactors, vibrotactor placement, vibrating frequency, stimuli duration, encoding signals and possible bandwidth used), type of outcome measures, and follow-up or retention and finally main results.

\section{Risk of bias assessment}

Two independent reviewers (KW and TP) independently assessed all included articles.

Suitability of randomisation and concealment of allocation, blinding of patients, healthcare providers, data collectors, and outcome assessors, and extent of loss to follow-up were determined by the two abovementioned reviewers using the PEDro (Physiotherapy Evidence Database) scale. According to de Morton, ${ }^{18}$ the PEDro scale is a valid tool to measure the methodological quality of clinical trials.

The risk of bias was assessed by the same authors (KW and TP) who performed the systematic search of literature. The two authors performed the assessment of methodological quality independently. Findings were compared for agreement but in case of disagreement, a third judgement was in all cases obtained by one of the other coauthors (SV). Agreement between the two reviewers concerning the scoring of methodological quality was determined by the Cohen's kappa. Cohen's kappa coefficient is a statistic measure for inter-rater agreement for qualitative items. In all cases, disagreements were resolved by discussion.

Scores were assigned based on meeting up with the criteria. A maximum possible score of 10 (low risk of bias) could be reached. Scores 6-10 were regarded as high-quality studies, 4-5 as fair quality and 3 or lower as poor quality. Studies with a risk of bias assessment score of 3 or less were considered poor and their contribution to results was weighted as half.

Evidence was graded according to EBRO (EvidenceBased Richtlijn[guideline]Ontwikkeling[development]) recommendations (table 3). ${ }^{19} \mathrm{EBRO}$ is an initiative of the Dutch Cochrane Centre and the Dutch Institute for Healthcare Improvement (CBO, http://www.cbo.nl), a member of the Guidelines International Network.

\section{Data analysis}

Since the average quality of the studies was poor, a quantitative or meta-analysis was not feasible. We tried 


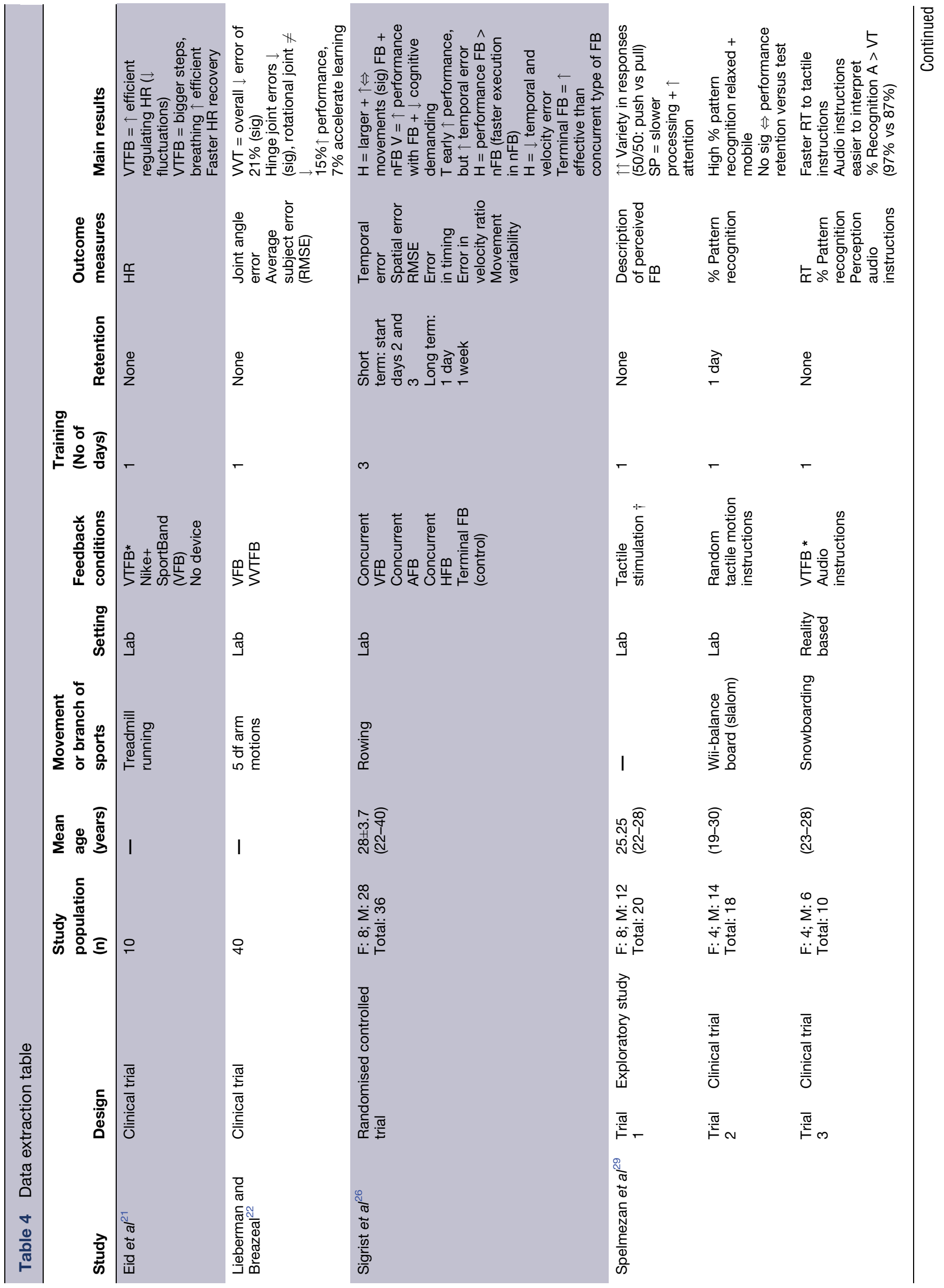




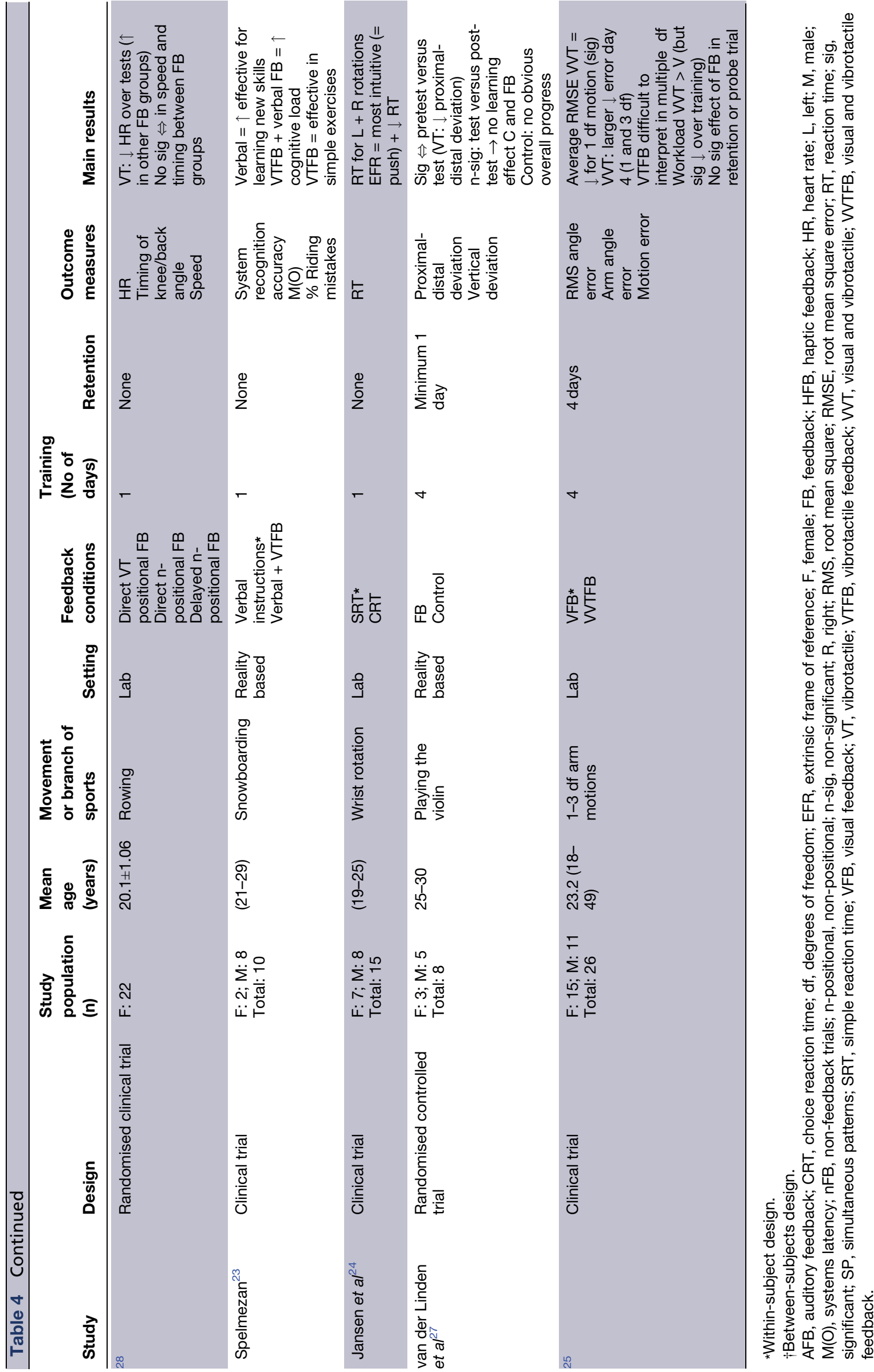


Table 5 Methodological scoring using the PEDro checklist and level of evidence of individual studies according to the CBO guidelines

\begin{tabular}{|c|c|c|c|c|c|c|c|c|c|c|c|c|c|c|c|}
\hline \multirow{2}{*}{\multicolumn{2}{|c|}{ Study }} & \multicolumn{12}{|l|}{ Items } & \multirow{2}{*}{$\begin{array}{l}\text { Methodological } \\
\text { quality }\end{array}$} & \multirow{2}{*}{$\begin{array}{l}\text { Level of } \\
\text { evidence }\end{array}$} \\
\hline & & 1* & 2 & 3 & 4 & 5 & 6 & 7 & 8 & 9 & 10 & 11 & Score ${ }^{* *}$ & & \\
\hline \multicolumn{2}{|l|}{ Eid et $a l^{21}$} & - & $?$ & $?$ & $?$ & - & - & - & - & + & - & - & $1 / 10$ & Poor & B \\
\hline \multicolumn{2}{|c|}{ Lieberman and Breazeal $^{22}$} & - & $?$ & $?$ & $?$ & + & $?$ & $?$ & + & + & + & + & $5 / 10$ & Fair & B \\
\hline \multicolumn{2}{|l|}{ Sigrist et $a l^{26}$} & + & + & $?$ & + & $?$ & $?$ & $?$ & + & + & + & + & $6 / 10$ & High & B \\
\hline \multirow[t]{3}{*}{ Spelmezan et $a l^{29}$} & $\begin{array}{l}\text { Study } \\
1\end{array}$ & - & + & $?$ & + & + & $?$ & $?$ & $?$ & + & - & - & $3 / 10$ & Poor & $\mathrm{C}$ \\
\hline & $\begin{array}{l}\text { Study } \\
2\end{array}$ & - & - & - & - & $?$ & $?$ & $?$ & + & + & + & + & $4 / 10$ & Fair & $\mathrm{C}$ \\
\hline & $\begin{array}{l}\text { Study } \\
3\end{array}$ & - & + & $?$ & - & $?$ & - & $?$ & + & + & + & + & $5 / 10$ & Fair & B \\
\hline \multicolumn{2}{|l|}{ Van Erp et $a l^{28}$} & $?$ & + & $?$ & + & $?$ & $?$ & $?$ & + & + & + & + & $6 / 10$ & High & $\mathrm{B} / \mathrm{A} 2$ \\
\hline \multicolumn{2}{|l|}{ Spelmezan ${ }^{23}$} & - & + & $?$ & - & - & + & + & + & + & + & + & $7 / 10$ & High & B \\
\hline \multicolumn{2}{|l|}{ Jansen et $a l^{24}$} & $?$ & - & - & + & $?$ & $?$ & $?$ & + & + & + & + & $5 / 10$ & Fair & B \\
\hline \multicolumn{2}{|l|}{ van der Linden et $a l^{27}$} & - & - & $?$ & + & $?$ & $?$ & $?$ & + & + & + & + & $5 / 10$ & Fair & B \\
\hline \multicolumn{2}{|l|}{25} & - & + & $?$ & + & $?$ & $?$ & $?$ & + & + & + & + & $6 / 10$ & High & B \\
\hline
\end{tabular}

\section{Scoring by the PEDro scale:}

$+=$ Yes

$-=$ No

? = Too little information or doubtable.

Legend:

1: Eligibility criteria were specified.

2: Subjects were randomly allocated to groups (in a crossover study, subjects were randomly allocated an order in which treatments were received).

3: Allocation was concealed.

4: The groups were similar at baseline regarding the most important prognostic indicators.

5: There was blinding of all subjects.

6: There was blinding of all therapists who administered the therapy.

7: There was blinding of all assessors who measured at least one key outcome.

8: Measures of at least one key outcome were obtained from more than $85 \%$ of the subjects initially allocated to groups.

9: All subjects for whom outcome measures were available received the treatment or control condition as allocated or, where this was not the case, data for at least one key outcome were analysed by 'intention to treat.'

10: The results of between-group statistical comparisons are reported for at least one key outcome.

11: The study provides both point measures and measures of variability for at least one key outcome.

\section{PEDro scores:}

High quality $=$ score $6-10$

Fair quality = score $4-5$

Poor quality $=$ score $\leq 3$

*Item 1 pertains for the external validity, thus is not considered for the final score.

$\star \star$ Poor quality $=$ PEDro score $\leq 3$.

CBO, Dutch Institute for Healthcare Improvement; PEDro, Physiotherapy Evidence Database.

to analyse the data from the included studies in a fashion as has been proposed by Saw et al. ${ }^{20}$ However, because of the diversity in outcome parameters and in objective data, we have not been able to perform such analysis. Therefore, the results of the individual studies will be presented qualitatively.

\section{RESULTS}

\section{Study selection}

The PRISMA flow diagram (figure 1) summarises our study selection process. The initial search yielded 312 results, of which 16 duplicates were deleted, leaving a total of 296 studies. The manual search and repeated search yielded a total of 25 extra studies, making a total of 321 studies that were selected for screening. From the initial 296 studies, 274 were excluded based on title and abstract. Subsequently, 38 studies were excluded based on full-text screening. Of the 25 studies obtained by the manual and repeated search performed on 30 March 2017, a total of 14 were excluded based on title and abstract, and 6 on the fulltext screening. Nine ${ }^{9}$ studies remained eligible for this review. 
The majority of the studies were excluded based on intervention and population, 63\% and 20\%, respectively. Twenty ${ }^{20}$ studies were excluded based on its study design and 24 based on outcome. Finally, three ${ }^{3}$ studies were excluded based on subject, eight ${ }^{8}$ studies on language and four ${ }^{4}$ studies were excluded based on age of the population. The majority of full-text exclusions were based on intervention.

\section{Study characteristics}

For each included study, the characteristics of data extraction are shown in table 4 . All nine ${ }^{9}$ studies were published in English and could be designated as clinical study designs with VTFB as (one of) the intervention(s). The majority of the studies involved simple clinical trials ${ }^{21-25}$ whereas two studies $^{26} \quad 27$ involved a randomised controlled trial. Only one study could be designated as a randomised clinical trial. ${ }^{28}$

The study of Spelmezan $e t a l^{29}$ consists of one exploratory clinical trial with an open response paradigm and two clinical trials, one of which was performed in a reality-based context. Two studies investigated the effects of VTFB during rowing, two during snowboarding, one focused on treadmill running and two during a simple arm movement. ${ }^{26}{ }^{28}$ One study investigated the effects of VTFB during an artistic skill. ${ }^{27}$

In two studies ${ }^{23} 27$ and in one of the trials of Spelmezan et $a l,{ }^{29}$ the experiments were done in a real rather than a laboratory setting.

VTFB was compared with other FB modalities in the majority of studies, except for the study of Jansen et $a l^{24}$ and in the first two trials of Spelmezan $e t$ al. ${ }^{29} \mathrm{In}$ the latter, VTFB was the only intervention. Outcome parameters differed between all studies. The main outcome parameters were joint angle error, root mean square error (RMSE), percentage of correct pattern recognition, reaction time and HR. Secondary and additional outcome parameters included temporal and spatial errors, timing errors and subjective description of perceived FB. In five studies, ${ }^{22} 23252629$ participants' subjective impression on the FB modalities was assessed using a questionnaire or interview. Duration of the intervention, that is, number of training days, ranged from 1 to 4 days. Only four studies included a retention phase ranging from a 1-day retention test to a 1 -week ${ }^{26}$ retention test.

\section{Risk of bias within studies}

Table 5 shows the methodological scoring and the assessment of the level of evidence.

We found a significant Cohen's kappa agreement between the two authors who performed the reviewing concerning the scoring of methodological quality $\left(\kappa_{\mathrm{w}}=0.983 ; \mathrm{p}<0.001\right)$. In $97 \%$ of the cases, both reviewers agreed. In all cases, disagreements were resolved by consensus, and if no consensus could be reached by the two authors (KW and TP) a third author was asked (SV). Each item contributes one point to the total PEDro score ranging from 0 to 10 points. Item 1 is not considered for the final score as it relates to external validity. Of the nine included studies, one had a final PEDro score of $1,{ }^{21}$ three $^{22}{ }^{2427}$ had a final score of 5, three ${ }^{25} 26^{28}$ had a final score of 6 and one $^{23}$ had a final score of 7 . The study of Spelmezan et $a l^{29}$ contains three trials of which one had a final score of 3 , one had a final score of 4 and the third had a final score of 5 . The overall quality of the included studies was fair to excellent, with the exception of the study of Eid et al (score 1) ${ }^{21}$ and the first trial of the Spelmezan et al's study (score 3). ${ }^{29}$ Most studies were rated with a lower score based on blinding and randomisation.

\section{Results of individual studies}

The effects of VTFB in regulating optimal training intensity

Eid et al studied the effectiveness of VT stimuli to adjust exercise intensity. ${ }^{21} \mathrm{~A}$ within-subject design was used to compare 'training with VTFB' with no device and one with a Nike+ SportBand, using HR as the main outcome parameter. Unfortunately, only one subject was presented, but based on VTFB was shown to be more efficient to regulate HR, showing fewer fluctuations and resulting in a smooth change in method by taking bigger steps and breathing more efficiently. A faster HR recovery was found in the VTFB condition.

VTFB in learning single and multiple degrees of freedom of arm movements and initiating wrist rotations

Lieberman and Breazeal ${ }^{22}$ and Bark et $a l^{25}$ studied whether VTFB, based on the push protocol and sensory saltation, accelerates learning of single and multiple degrees of freedom (df) arm movements and reduces motion errors. Lieberman ${ }^{22}$ compared visual FB $(n=20)$ with the combination of visual FB and VTFB $(n=20)$. They found that the addition of VTFB to motor training induces significant change in performance: a reduction in real-time errors by up to $27 \%$ and an improvement of learning rate by up to $23 \%$; with a steady state in learning errors by $27 \%$ over flexion joints. Subjects with the combination FB showed a significant higher level of correcting their motions in comparison to those without TCB. No significant loss of comfort through the addition of the wearable has been reported by the subjects.

In line with the studies of Lieberman et al, ${ }^{22}$ Bark et $a l^{25}$ used a within-subject design to compare visual FB with the combination of visual FB and VTFB. They reported that RMSE was significantly lower for the combination group $\left(15.7^{\circ} \pm 6.5^{\circ}\right)$ in comparison to the visual only group $\left(17.1^{\circ} \pm 6.4^{\circ}\right)$ for learning $1 \mathrm{df}$ motion, but not for 2 or $3 \mathrm{df}$ motions. In both groups, motion errors decreased over the 4 days of training but no significant effect on joint angle error was found during probe or retention. 
Finally, Jansen et al studied whether VTFB was useful in initiating wrist rotations and which stimulus location provided the fastest reaction time. ${ }^{24}$ They concluded that an extrinsic reference frame in which the vibratory signal 'pulls' the hand in the desired direction is most intuitive.

\section{Effectiveness of VTFB in a real sports context}

Spelmezan et al conducted a within-subject study in snowboarding in which traditional verbal instructions in combination with VTFB were compared with traditional verbal $\mathrm{FB}$ only. ${ }^{23}$ Verbal instructions in the morning resulted in fewer mistakes compared with VTFB $(p=0.044)$, whereas the opposite was true for trials in the afternoon $(p=0.014)$. Verbal instructions outweighed VTFB when practising new tasks. Such results are in line with another study of Spelmezan et al in which snowboarders were provided with verbal $\mathrm{FB}$ instructions with or without $\mathrm{VTFB}^{29} \mathrm{~A}$ faster response time has been found for VTFB in comparison with verbal $\mathrm{FB}(\mathrm{p}=0.01)$.

The effectiveness of VTFB in learning a rowing task was studied by Van Erp et al and Sigrist $e t$ al. ${ }^{26}{ }^{28}$ Van Erp et $a l^{28}$ compared direct positional VTFB with direct non-positional verbal FB and delayed non-positional verbal feedback. Neither group showed a learning effect. The VT group showed a significant $(p<0.02)$ decrease in HR over tests but no difference was found between groups regarding timing and speed. Sigrist et $a l^{26}$ compared VTFB with verbal $\mathrm{FB}$ and auditory FB with a self-controlled terminal FB. Terminal self-controlled FB was found to be superior to all types of concurrent AFB. Furthermore, a significant decrease in RMSE, temporal error and error in velocity ratio was found from baseline to retention indicating that VTFB is effective in enhancing temporal aspects, rather than spatial aspects of movement. Again, a guidance effect could be found, that is, decrease in performance in non-FB conditions. ${ }^{30}$

\section{DISCUSSION}

The aim of the present review was to provide a more detailed insight in the scientific literature concerning VTFB as a tool for sports performance enhancement. Only nine studies, based on the methodological criteria set, were eligible for this review. The quality of the studies was fair but the level of evidence was suboptimal. We conclude that, although VTFB has been shown to be effective under some conditions, evidence for its use as sports performance enhancement remains scarce and challenging. ${ }^{10} 1131$

\section{Summary of evidence}

VTFB has been used in navigation, ${ }^{32}$ aviation ${ }^{33}$ and in automotive. ${ }^{34}$ It also found its way in virtual reality, gaming and in art. ${ }^{35}{ }^{36}$ In healthcare, VTFB has been used as a navigation tool in the visually impaired subjects, as sensory substitution in the rehabilitation of patients with stroke, and in vestibular impaired subjects where it has been used as sensory augmentation. ${ }^{37}$ To the best of our knowledge, its use in sports practice is very limited. A reason for this is not easy to provide but the limited number of scientific studies in concordance with the broad range of outcome parameters and variability of the results in laboratory setting may be the major cause of the lack of use in sports practice.

Out of the nine studies eligible for final interpretation, only the studies of Spelmezan et al and van der Linden $e t a l^{23} 2729$ addressed VTFB in a reality-based context. For instance, van der Linden et al implemented the use of VTFB in real training situations for artists (violin players) whereas Spelmezan used snowboarders as study population. Subjects in the later study, however, stated that the VTFB instructions hindered normal movement because VT instructions were randomly provided in time and the placement on the body/limbs was reported to be frustrating. This might be one of the major reasons for athletes not to use VTFB devices on their body/limbs even if it might help them to improve performance. These findings are in contrast with the findings of Lieberman et al, ${ }^{22}$ who reported no loss of comfort during the application of VTFB. The use of devices that differ in size, weight and position could play a major role in the acceptance of VTFB in sports practice. Future studies should therefore be designed such that VTFB does not interfere with normal movement by providing VT instructions on suitable moments of VTFB.

There is some evidence that VTFB is effective in regulating exercise intensity during physical performance by keeping HR within aerobic limits. ${ }^{18}$ By applying VTFB, athletes could, based on their maximal $\mathrm{HR}$, be guided by tactile information to perform at a less intense level and thus exercise more efficiently. Exercising with VTFB resulted in both short term and long term, in a decrease in HR. ${ }^{22}$ The athletes in their study who received TFB (ie, direct and positional) showed a decreased HR in the post-test as compared with the pretest whereas the athletes who did not get TFB showed an increase in HR. A plausible explanation for this HR effect was that athletes without VTFB were less efficient in maintaining motor skills and more than necessary adjustments in motor control were necessary. Therefore, it is reasonable to assume that VTFB is effective in improving temporal movement aspects, but not spatial errors. ${ }^{26}$ These findings support the results of previous studies. ${ }^{38} 39$ Lastly, there is moderate evidence that VTFB instructions could support the athlete in practising and fine-tuning a previously learnt movement. A second explanation for an over-the-day decrease in HR is that VTFB is less mental demanding. ${ }^{25}$ To date, no studies are present that support this idea. 
Some studies used joint angle errors as the main outcome parameter. ${ }^{19} 22$ This is somewhat surprising since joint angle is not necessarily the most important outcome parameter for motor performance, and as such, athletic performance. Moreover, during a motor task, several joint angles should be taken into account, that when applying VTFB should provide simultaneous and multiple VTFB signals. ${ }^{23} 2527$ The major drawback, however, of some studies using VTFB as error signal is the lack of implementing an order or sequence in signals. ${ }^{22} 28$ Subjects are continuously provided with FB signals which lead to overcorrection of motor tasks/movements. Only a few scientific studies that used VTFB for motor task correction used more accurate and timed signals. ${ }^{21}$ 25-27

\section{Limitations of the current review}

The quality and diversity of the studies made it impossible for us to do quantitative analysis of the results. The risk of bias of individual studies was overall moderate to fair and because randomisation was inadequate or lacking in the majority of trials, level of evidence was moderate to weak. Furthermore, poor and incomplete reporting of study designs, patient populations and interventions made the interpretation and synthesis of the included studies much more difficult and may have contributed to publication bias. Therefore, generalisation of the results to athletes is difficult.

Finally, the main limitation of this study is that the context of VTFB and comparison between different FB modalities and outcome measures for performance were not the same across the studies, making a direct comparison between the outcome of these studies difficult.

\section{CONCLUSIONS}

Our review suggests that VTFB, which has been used as application in medical and non-medical-based contexts (ie, patient groups, automotive, and so on), lacks scientific support for enhancement of sports performance outcomes. The inconsistency of findings and moderate level of support, as reported in the present study, hardly provide evidence to support the suspected role. More basic and applied studies are necessary to warrant its use in a sports context.

Contributors The study was planned by EvB, SV and ST. KW and TP conducted the literature search whereas SV was used as author in case of disagreements during selection. EvB and SV wrote the initial draft of the manuscript. EvB, SV, WS and ST discussed and revised the manuscript together and gave final approval for publication.

Competing interests None declared.

Provenance and peer review Not commissioned; externally peer reviewed.

Open Access This is an Open Access article distributed in accordance with the Creative Commons Attribution Non Commercial (CC BY-NC 4.0) license, which permits others to distribute, remix, adapt, build upon this work non- commercially, and license their derivative works on different terms, provided the original work is properly cited and the use is non-commercial. See: http:// creativecommons.org/licenses/by-nc/4.0/

Correction notice This paper has been amended since it was published Online First. Owing to a scripting error, some of the publisher names in the references were replaced with 'BMJ Publishing Group'. This only affected the full text version, not the PDF. We have since corrected these errors and the correct publishers have been inserted into the references.

(c) Article author(s) (or their employer(s) unless otherwise stated in the text of the article) 2017. All rights reserved. No commercial use is permitted unless otherwise expressly granted.

\section{REFERENCES}

1. Schmidt RA, Wrisberg CA. Motor learning and performance: a situation-based learning approach: human kinetics, 2008.

2. Utley A, Astill S. Motor control, learning and development. Int J Sports Sci Coach 2008;3:297-9.

3. Schönauer C, Fukushi K, Olwal A et al, eds. Multimodal motion guidance: techniques for adaptive and dynamic feedback Proceedings of the 14th ACM International Conference on Multimodal Interaction: ACM, 2012.

4. Minogue J, Jones MG. Haptics in Education: Exploring an Untapped Sensory Modality. Rev Educ Res 2006;76:317-48.

5. Sigrist R, Rauter G, Riener R, et al. Augmented visual, auditory, haptic, and multimodal feedback in motor learning: a review. Psychon Bull Rev 2013;20:21-53.

6. El Saddik A, Orozco M, Eid M, et al. Haptics: general principles Haptics technologies. Springer, 2011.

7. Hale KS, Stanney KM. Deriving haptic design guidelines from human physiological, psychophysical, and neurological foundations. IEEE Comput Graph Appl 2004;24:33-9.

8. Lauber B, Keller M. Improving motor performance: selected aspects of augmented feedback in exercise and health. Eur J Sport Sci 2014;14:36-43.

9. Schätzle S, Ende T, Wüsthoff T et al, eds . Vibrotac: an ergonomic and versatile usable vibrotactile feedback device. RO-MAN: 2010 IEEE, 2010.

10. Alahakone AU, Senanayake S, Arosha M, eds. Vibrotactile feedback systems: current trends in rehabilitation, sports and information display. Advanced Intelligent Mechatronics, 2009 AIM 2009 IEEE/ ASME International Conference on 2009: IEEE, 2009.

11. Choi S, Kuchenbecker KJ. Vibrotactile display: Perception, technology, and applications. Proceedingsof the IEEE. 2013;101:2093-104.

12. Gray R. Multisensory Information in the Control of Complex Motor Actions. Curr Dir Psychol Sci 2008;17:244-8.

13. Amemiya $T$, Ando $H$, Maeda $T$. Lead-me interface for a pulling sensation from hand-held devices. ACM Trans Appl Percept 2008;5:1-17.

14. Spelmezan D, Ziefle M. A language of tactile motion instructions for physical activities. RWTH Aachen University, 2011.

15. Moher DLA, Tetzlaff J, Altman DG. Preferred Reporting Items for Systematic Reviews and Me.Analyses: The PRISMA Statement, 2009. http://prisma-statementorg/.

16. Pressley M, Ghatala ES. Metacognitive benefits of taking a test for children and young adolescents. J Exp Child Psychol 1989;47:430-50.

17. Rodrigue KM, Kennedy KM, Raz N. Aging and longitudinal change in perceptual-motor skill acquisition in healthy adults. J Gerontol B Psychol Sci Soc Sci 2005;60:P174-P181.

18. de Morton NA. The PEDro scale is a valid measure of the methodological quality of clinical trials: a demographic study. Aust $J$ Physiother 2009;55:129-33.

19. Keus SH, Bloem BR, Hendriks EJ, et al. Practice Recommendations Development G. Evidence-based analysis of physical therapy in Parkinson's disease with recommendations for practice and research. Mov Disord 2007;22:451-60.

20. Saw AE, Main LC, Gastin PB. Monitoring the athlete training response: subjective self-reported measures trump commonly used objective measures: a systematic review. $\mathrm{Br} J$ Sports Med 2016:50:281-91.

21. Eid M, Saad U, Afzal U, eds. A real time vibrotactile biofeedback system for optimizing athlete training. Audio Visual environments and Games (Have), 2013 IEEE International Symposium on 2013. IEEE.

22. Lieberman J, Breazeal C. TIKL: development of a wearable vibrotactile feedback suit for improved human motor learning. IEEE Transactions on Robotics 2007;23:919-26. 
23. Spelmezan $D$, ed An investigation into the use of tactile instructions in snowboarding. Proceedings of the 14th international conference on Human-computer interaction with mobile devices and services. ACM;2012.

24. Jansen C, Oving A, van Veen H-J. eds. Vibrotactile Movement Initiation.Proceedings of Eurohaptics. Citeseer, 2004.

25. Bark K, Hyman E, Tan F, et al. Effects of Vibrotactile feedback on human learning of arm motions. IEEE Trans Neural Syst Rehabil Eng 2015;23:51-63.

26. Sigrist R, Rauter G, Riener R, et al. Terminal feedback outperforms concurrent visual, auditory, and haptic feedback in learning a complex rowing-type task. J Mot Behav 2013;45:455-72.

27. van der Linden J, Schoonderwaldt E, Bird J, et al. Musicjacketcombining motion capture and vibrotactile feedback to teach violin bowing. IEEE Trans Instrum Meas 2011;60:104-13.

28. Van Erp JB, Saturday I, Jansen C, eds. Application of tactile displays in sports: where to, how and when to move. Proc Eurohaptics. Citeseer;2006.

29. Spelmezan D, Jacobs $M$, Hilgers $A$, eds. Tactile motion instructions for physical activities. Proceedings of the SIGCHI conference on human factors in computing systems. ACM, 2009.

30. Salmoni AW, Schmidt RA, Walter CB. Knowledge of results and motor learning: a review and critical reappraisal. Psychol Bull 1984;95:355-86.
31. Shull PB, Damian DD. Haptic wearables as sensory replacement, sensory augmentation and trainer-a review. J Neuroeng Rehabil 2015:12 1.

32. Erp JBFV, Veen HAHCV, Jansen $\mathrm{C}$, et al. Waypoint navigation with a vibrotactile waist belt. ACM Trans Appl Percept 2005;2:106-17.

33. Rupert AH. Tactile situation awareness system: proprioceptive prostheses for sensory deficiencies. Aviat Space Environ Med 2000;71(Suppl 9):A92-9.

34. Scott JJ, Gray R. A comparison of tactile, visual, and auditory warnings for rear-end collision prevention in simulated driving. Hum Factors 2008;50:264-75.

35. Feeling the beat where it counts: fostering multi-limb rhythm skills with the haptic drum kit. In: Holland S, Bouwer AJ, Dalgelish M, eds. Proceedings of the fourth international conference on Tangible, embedded, and embodied interaction: ACM, 2010.

36. Mobile music touch: mobile tactile stimulation for passive learning. In: Huang K, Starner T, Do E, eds. Proc SIGCHI Conf Hum Factor Comput Syst : ACM, 2010

37. Wall C, Weinberg MS, Schmidt PB, et al. Balance prosthesis based on micromechanical sensors using vibrotactile feedback of tilt. IEEE Trans Biomed Eng 2001;48:1153-61.

38. Crespo LM, Reinkensmeyer DJ. Haptic guidance can enhance Motor Learning of a steering Task. J Mot Behav 2008;40:545-57.

39. Haptic interfaces for virtual environment and teleoperator systems, 2002 haptics 2002 proceedings 10th symposium on; 2002: IEEE. In: Feygin D, Keehner M, Tendick F, eds. Haptic quidance: Experimental evaluation of a haptic training method for a perceptual motor skill. 